\title{
Pattern Formation and Functionality in Swarm Models
}

\author{
Erik M. Rauch ${ }^{1}$, Mark M. Millonas ${ }^{2}$ and Dante R. Chialvo ${ }^{3}$ \\ ${ }^{1}$ Yale University, P.O. Box 202200 Yale Station, New Haven, CT 06520 \\ ${ }^{2}$ Theoretical Division and CNLS, MS B258 Los Alamos National Laboratory, \\ Los Alamos, NM 87545 \\ ${ }^{3}$ Division of Neural Systems, Memory and Aging, University of Arizona, Tucson AZ 85724
}

October 26, 2018

\begin{abstract}
We explore a simplified class of models we call swarms, which are inspired by the collective behavior of social insects. We perform a mean-field stability analysis and perform numerical simulations of the model. Several interesting types of behavior emerge in the vicinity of a second-order phase transition in the model, including the formation of stable lines of traffic flow, and memory reconstitution and bootstrapping. In addition to providing an understanding of certain classes of biological behavior, these models bear a generic resemblance to a number of pattern formation processes in the physical sciences.
\end{abstract}

\section{The Model}

The self-organization of patterns of flow in social insect swarms is a beautiful example of how intelligent and efficient behavior of the whole can be achieved even in the absence of any particular intelligence or forethought of the individuals. [1] Indeed, such patterns can have functionality even without the awareness of the individual entities themselves. A study of the essential elements of swarm dynamics provides an understanding of such behaviors, 
and is our principal goal in this letter. A secondary, broader goal is to study the generic behavior of a kind of stochastic particle-field system in which the motion of the "particles" both changes the field and is affected by the field. There are a number of systems like this in the physical sciences. We particularly have in mind systems where the relaxation time scales of the field are slow in comparison to the motion of the particles and this is what makes the present work unique and interesting.

Consider the following scenario. Suppose particles move in a field $\sigma(\mathbf{x})$ that field corresponds to an energy $U[\sigma(\mathbf{x})]=U(\mathbf{x})$ so that each particle experiences a force due to the field of $\mathbf{F}=-\nabla U(\mathbf{x})$. Suppose also that the particles are subject to random perturbations so that their motion is only statistically in the direction of $\mathbf{F}$. We could describe their motion by the stochastic (Langevin) type equation

$$
\ddot{\mathbf{x}}+\Gamma \dot{\mathbf{x}}+\nabla U[\sigma(\mathbf{x})]=\mathbf{f}(t) .
$$

where $\mathbf{f}(t)$ is Gaussian white noise with $<\mathbf{f}(t)>=\mathbf{0}$ and $<\mathbf{f}(t) \cdot \mathbf{f}(s)>=$ $(1 / \beta) \mathbf{1} \delta(t-s)$. The parameter $\Gamma$ is a friction coefficient. Obviously for social insects this term is not to be taken literally. The "friction" in such cases merely parameterizes the tendency of the organism-particles to continue in a given direction. A smaller "friction" thus means that the organism's velocity vector is correlated over longer times. In physical systems this parameter may have a more literal meaning.

The noise strength is $1 / \beta$. Thus $\beta$, which we call the osmotropotaxic sensitivity particle follow the gradient of the field $\mathbf{F}(\mathbf{x})=-\nabla U(\mathbf{x})=-U^{\prime}[\sigma(\mathbf{x})] \nabla \sigma(\mathbf{x})$. If $\beta$ is large the predominant force felt by the particles is $\mathbf{F}$, and they follow $\mathbf{F}$ more accurately. If $\beta$ is small the noise becomes more important and the organism-particles follow the field gradient with less certainty. It is necessary to assume probabilistic laws in order to reproduce the behaviors observed experimentally, and the osmotropotaxic sensitivity can be, and has been, observed experimentally. [5, 6, 7] As we will see, the degree of randomness plays a very important role in the functionality of the swarm. Most probabably it is hardwired into the sensory apparatus of the organism.

Up to this point we have simply described the noisy motion of particles in some "energy landscape". The principal element of interest here is that the

\footnotetext{
${ }^{1}$ Osmotropotaxis is pheromone gradient following, as explained in greater detail in 边, 回, 四
} 
energy landscape will be allowed to evolve in response to the noisy motion of the particles. We will choose one of the simplest possible models for how this occurs. [9, 10, 11] The presence of a particle at $\mathbf{x}$ will "deposit" an amount of field $\eta$ per unit time. For insect swarms $\sigma(\mathbf{x})$ is to be interpreted as the pheromonal field density 12 at $\mathbf{x}$. An amount of field $\kappa \sigma(\mathbf{x})$ will "decay" per unit time. The field might also diffuse in space with diffusion constant $D$ such that the field will evolve according to

$$
\frac{\partial \sigma(\mathbf{x})}{\partial t}=D \nabla^{2} \sigma(\mathbf{x})+\eta \rho(\mathbf{x})-\kappa \sigma(\mathbf{x})
$$

where $\rho(\mathbf{x})$ is the particle density at $\mathbf{x}$. When the particles are social insects this equation describes the evolution of pheromonal field $\sigma$ laid down by the organisms as they walk, and the parameter $\kappa$ is an evaporation rate.

The only thing which has been left unspecified here is $U[\sigma]$. In this letter we use

$$
U[\sigma]=-\ln \left(1+\frac{\sigma}{1+\delta \sigma}\right) .
$$

The use of this "field energy function" has already been justified elsewhere, 9 , 10, 11 where it has been explained at length how this function is derived from the experimentally observed behavior of real ants. Other energy functions can be used for different physical situations, and the general framework of this model is capable of supporting many different types of systems.

Here we call $1 / \delta$ the capacity. This models the fact that an actual ant's response to additional concentration of pheromone decreases somewhat at high concentrations. This gives rise to a peaked function for the average time an ant will stay on a line of ant traffic as the concentration of pheromone is varied - a fact which has been repeatedly observed experimentally[13, 14, 15]. This is perhaps significant in light of one of the results presented here: trails and networks do not spontaneously form in the absence of this saturation effect. One reasonable physiological explanation for this effect is that the ant's sensing organs become saturated: since each antenna has only a finite number of pheromone receptor sites, the antennae are effectively jammed at high concentration, and the response to gradients can be expected to grow less pronounced.

The model described above was constructed precisely because it approximately reproduces the "microscopic" behavior of individual ants as observed in the laboratory. [8, 3, 4, [] Notice that we have resisted the temptation to 
write a Fokker-Planck equation for $\rho(\mathbf{p}, \mathbf{x}, t)$ from Eq. 1. This is to emphasize that we do not want to consider the continous limit, but rather the case where internal fluctuations are significant. Since the field $\sigma$ corresponds to the pheromone which, unlike the ant density $\rho$, is composed of a macroscopically large number of particles, we can use the continuous Equation 2 to describe it. We have expressed the model in this particularly suggestive form to emphasize its physical aspects, and its generic relationship to other physical systems. We will not expand on this point here, but will merely indicate the generic resemblence of this model to problems such as anomalous ionic diffusion in polymeric materials, stochastic growth processes, the evolution of river basins, and other types of complex physical systems that incorporate mobile elements and an evolving substrate of some kind. 16.

\section{Analysis}

Since a detailed mathematical analysis of this type of system has already been made [9, 10], we will confine ourselves here to a brief statement of the pertinent results.

We analyze the case where the relaxation of the field $\sigma(\mathbf{x})$ is very slow in comparison to the relaxation time of the particle density $\rho(\mathbf{x})$. In this case the field is said to slave 18 to the particle denisty, and the particle density can be removed from the picture in the following way. Since the field changes very little on these time scales we will assume it is constant as the particles equilibrate in the energy landscape $U[\sigma(\mathbf{x})]$. It is easy to show that the equilibrium distribution of particles evolves to

$$
\rho_{e}[\sigma(\mathbf{x})]=\frac{\exp (-\beta U[\sigma(\mathbf{x})])}{\int d^{D} \mathbf{y} \exp (-\beta U[\sigma(\mathbf{y})])}
$$

Elimination of the particle variables is made possible by substituting this expression into Eq. 2, so that

$$
\frac{\partial \sigma(\mathbf{x}, t)}{\partial t}=\frac{\eta N \exp (-\beta U[\sigma(\mathbf{x}, t)])}{\int d^{D} \mathbf{y} \exp (-\beta U[\sigma(\mathbf{y}, t)])}-\kappa \sigma(\mathbf{x}, t),
$$

where $N$ is the total number of particles, and where we neglect diffusion and will not consider it futher in the present letter. For our present purpose we will only be interested in the stability of the uniform phase of the system, 
$\sigma(\mathbf{x}, t)=\sigma(t)$, which we will treat by mean-field theory. The above equation has a mean-field solution of $\sigma_{0}=N \eta / V \kappa$, where $V=\int d^{D} \mathbf{y}$.

The stability of this solution is found by expanding about the solution with $\sigma(\mathbf{x}, t)=\sigma_{0}+\delta \sigma(\mathbf{x}, t)$. This leads to the mean-field stability criterion

$$
\beta \sigma_{0} U^{\prime}\left[\sigma_{0}\right]+1>0
$$

This is the most important theoretical result, and it gives the location of the second-order phase transition, that is, the location of the boundary separating totally random behavior from ordered behavior of varying types in the physiological phase space. As shown in [9, 10], ordered behavior sets in when this criterion is broken. This criterion is true for any energy function $U[\sigma]$, and allows us to calculate the physiological phase boundaries in every case.

For the particular behavioral energy function used here (Eq. 3) the transition lies along the curve

$$
\beta_{c}\left(\delta, \sigma_{0}\right)=1+1 / \sigma_{0}+2 \delta+\delta \sigma_{0}+\delta^{2} \sigma_{0} .
$$

The symmetry which allows for a mean-field type solution for the location of the transition line case (detailed balance) is maintained up to the point of the transition and is effectively spontaneously broken at that point. This means the points of transition from disorder to order can be determined theoretically, but not the resulting patterns, which must be determined via simulations or further theoretical analysis.

In addition to being the major landmark in the physiological parameter space, we believe both on general grounds, and because of the results presented in the next sections, that the location of this line has significant behavioral implications. Clearly it is important that ordered behavior exists so that ordered patterns of flow can form, but just as importantly, the behavior should not be too rigid and ordered since fluctuation and instabilities might increase the flexibility of response of the mass action. Thus, if the dynamics are such that there can be significant fluctuations in the patterns of mass action, the swarms could better respond to a changing environment. We might conclude that a "good" place to be would be in the order region, but near to the transition line in such a way as to optimize the conflicting tendencies of controlled order behaviors versus flexible random behavior. We will also see in the next sections that large fluctuation actually serves to stabilize some of the important patterns of collective behavior of the system. 


\section{$3 \quad$ Numerics}

Equations 1, 2 and describe the system, but for the purposes of simulation it is necessary to introduce some discretization of space and time, and to translate the noisy behavioral function observed experimentally into transition rules on this discrete space [9]. These discrete rules are merely tools for the approximation of a continuous model, and other discretizations are possible.

The agent (referred to as an "ant") will be allowed to move from site to site on a square lattice. We allow each ant to take one step on the lattice of points (cells) at each time step. As a result of discretizing the space, an individual ant at each time step finds itself in one of these cells, and its sensory input is the concentration of pheromone in its own cell and each of the eight neighboring cells. In addition, each ant leaves a constant amount of pheromone at the node in which it is located at every time step. This pheromone decays (is reduced by a certain percentage) at each time step. Toroidial boundary conditions are imposed on the lattice to remove, as much as possible, any boundary effects. The transition rates from cell $i$ to cell $j$ are proportional to $W \propto \exp \left(-\beta\left(U\left[\sigma_{j}\right]\right)\right.$. The normalized transition probabilities on the lattice to go from cell $k$ to cell $i$ are then given by

$$
P_{i k}=\frac{W\left(\sigma_{i}\right) w\left(\Delta_{i}\right)}{\sum_{j / k} W\left(\sigma_{j}\right) w\left(\Delta_{j}\right)}
$$

where the notation $\Sigma_{j} / k$ indicates the sum over all the cells $j$ which are in the local neighborhood of $k . \Delta_{i}$ measures the magnitude of the difference in orientation (direction) to the previous direction the last time the ant moved. Since we are using a neighborhood composed of the cell and its eight neighbors on a square lattice, $\Delta_{i}$ can take only the discrete values $0-4$, and it is sufficient to assign a number $w_{i}$ for each of these changes of direction. Here we used weights of (same direction) $W_{0}=1$, and $w_{1}=1 / 2, w_{2}=1 / 8$, $w_{3}=1 / 12$ and $w_{4}=1 / 50$ (u-turn). Once the parameters $\beta, \delta$ and $w_{i}$ are set a large number of ants can be placed on the lattice at random positions, the movement of each ant can be determined randomly taken from the distribution given by $P_{i k}$. We usually take the initial condition of the pheromone to be zero at every point on the lattice. Every time step each ant leaves a quantity $\eta$ (here $\eta=0.05$ ) of pheromone in each cell, and the total amount of pheromone $\sigma_{i}$ in each cell is decreased at a rate $\kappa$ (here $\kappa=0.015$ ) at the 
end of each time step. The evolution of the system is simulated numerically and the pattern of lattice sites that contain ants is displayed in the figures.

\section{The Physiological Phase-Plot}

We studied a wide range of scent laying and decay rates, as well as different densities of ants. This is the simplest local, memoryless, homogeneous and isotropic model which leads to trail forming that we know of, and the formation of trails and networks of ant traffic is not imposed by any special boundary conditions, lattice topology, or additional behavioral rules. The required behavioral elements are stochastic, nonlinear response of an ant to the scent, and a directional bias. Furthermore the parameters of the system need to be tuned somewhat to the appropriate region, and not any nonlinear rule of the above type will do. If the nonlinear response or the directional bias are removed no lines form, and lines that are already formed do not persist.

Well defined trails form in the region above the phase transition line (ordered phase), but near the transition from disorder. Further away from the order-disorder line the clumping tendency overcomes the directional bias, and no lines form.

In every run observed within the part of the parameter space indicated in Figure 4, a final system of trails emerged and survived as long as the simulation was run (Fig. 1). The self-organization of regular patterns of movement of the ants occurs in two stages: an initial condensation phase, which occurs during approximately the first 500 time steps, and a simplification phase. In the first stage a network of trails forms within a few hundred time steps. The trails at first typically form a network of many branches. All of the branches initially appear stable, but after several hundred time steps, some abruptly disappear, decreasing the length of the network.

In this second stage the network of ant traffic resembles qualitatively some network type experiments done with real ants [7] and the network theory of [9], (and the basic discussion of the properties of these networks given there holds). The ants can neglect a branch by chance, and if the fluctuation in the density of the ants over the branch happens to be great enough, the neglect builds on itself and the trail disappears. As a result, the complexity of the network tends to decrease with time until a final, stable network is reached. 
The final line in all runs has been a loop (sometimes with sub-loops). Most often, the trail exploits the periodic boundary conditions by forming a more-or-less straight line that wraps around the torus. This of course reflects the situation that a line "free end" is clearly unstable due to the directional bias. That lines which feed back on themselves invariably form is thus not surprising. It is interesting to note here the qualitative similarity of these loops to autocatalytic sets of chemical reactions. 19, 20 The instability of the free ends just illustrates the sensitivity to boundary conditions when the information flows in an active way. The separate issues of how these basic structures are incorporated into a larger colony context will be taken up elsewhere, and we believe they deserve further mathematical analysis.

The main conclusion to be drawn here is that simple osmotropotaxic scent following of the very simple kind described above is no only sufficient to allow for trail following behavior as shown in [3, th, but sufficient to produce evolution of complex pattern of organized flow of social insect traffic all by itself.

\section{Consolidation, Memory, and Bootstrapping}

Since the self-organizing properties of the swarm are instability driven, the structures that form have some very interesting properties with respect to large perturbations. We performed the following experiment: the system was tuned to a region of the phase plane where lines form, and a stable network of traffic in a straight line was set up. Then the noise level was increased so that system is tuned below the transition line $\left(\beta<\beta_{c}\right)$. One observes that the ants fall away from their orderly patterns and immediately start executing random walks on the lattice. As a result the pheromone distribution starts to fluctuate and become more and more random. If $\beta$ is then tuned back to its original value at some time later the line will eventually reform with little or no change. This occurs even if the randomization is allowed to proceed to the point that the pheromonal field is almost totally randomized.

Fig. 3 is shows a measure of how much of the original pattern in the pheromonal field is left over time as the ants execute a random walk for a certain time and then go back to nonlinear scent response. We define $\pi$ as the number of lattice sites where the concentration of pheromone is above the average for the grid in both the original pattern and the current field, as 
a percentage of the number of current sites with above average pheromone. Thus $\pi$ is a measure of the overlap of the current pattern with the original pattern. The figure plots $\pi$ for different durations of the random walk, and shows the stable patterns that re-form after the nonlinear scent response is turned back on. For times up to about twice the decay time $\tau=1 / \kappa$ even small, virtually undetectable memory effects of the field can be amplified causing the patterns to reform without significant changes. As shown in Fig. 3 , as much as $90 \%$ of the pattern can be erased, and entirely reconstituted later.

To further show the adaptability of the swarm in certain regions of the parameter space, the following experiment was performed. The system was tuned to a region of $\beta-\delta$ parameter space where trails never form, and the motion of the ants remains random. A weak "bootstrapping" trail was then added to the grid, the pheromone density of which was on the order of some of the larger random fluctuations in the field. In a region slightly below the phase transition, this causes the swarm to organize and amplify the trail, in spite of the fact that a network would not have formed spontaneously. Fig. 4 plots a measure of pheromone concentration $v$, which is the ratio of lattice sites with below-average pheromone concentration to those with above-average concentration. Three runs are shown; one where no bootstrapping trail is added; another in which a bootstrapping trail causes the swarm to shift into the trail, and lastly, an attempt at bootstrapping which failed because the swarm was too far from the phase transition line. We emphasize that this behavior appears below, but near enough to the phase transition line.

Memory reconsolidation and bootstrapping are two seemingly conflicting functional abilities which appear in the vicinity to the phase transition line. Firstly, these organized patterns of behavior are really quite stable with respect to large perturbation,s which might have an obvious usefulness to operation in a changing and unpredictable environment. Secondly, because the patterns are due to the formation of an initially weak stable cooperative structure, the swarm can act as an information amplifier, and even a weak external perturbation (such as the location of a food source by a single ant) might lead to a significant response. Thus swarms posses both a long memory and the ability to learn. 


\section{Conclusions}

As shown in Fig. 2, lines of traffic form near the second-order phase transition line. In this region there is a cooperative effect between the fluctuations and the ordering effects. Much below the transition line the fluctuations are too great, and no cooperative structures form. However, too far above the transition line the fluctuations are suppressed, and order dominates overwhelmingly. This means that the ants are basically induced to "turn around" with sufficient probability that only patches form. The role of such "U-turns", has been investigated experimentally and theoretically in [21], where it was also suggested that they might play an important role in the self-organization of ant traffic.

The region in which the lines form represents a cooperative effect between the fluctuations and the ordering behavior. The formation of lines can also be understood in terms of the theory in [9, 10]. In the region of the lattice where there are lines, the clumping effect is both unstable in the transverse direction and stable in the lateral direction of the particle motion, and it is this fact which gives rise to the formation of stable lines of traffic. The result is a fairly robust region of line formation. Motion in this region can perhaps be likened to motion in the liquid crystal phase of matter, or the reptation of polymer chains in polymer melts, in which the order along one axis is frozen in, while along another motion can occur freely.

Since information (in the form of the orderly movement of ants from one place to another) can be said to flow only in the region of stable line formation, which lies above the disordered region and below the very ordered region, these results might mistakenly be thought similar to the "meta-theory" of "complexity at the edge of chaos" which asserts that complex behavior emerges in the vicinity of a marginally stable state. 222, 23, 24, 25] However we point out that the system, as far as we know, does not "self-organize" to this region unless it may be said that on the evolutionary time scale the biological organisms found such behavior adaptive. In addition there is not an "edge" but rather a large robust region where the most complex structures form. Lastly we do not believe that the reason for this type of behavior has anything to do with the very speculative ones which are sometimes suggested. It is quite natural that complex behaviors resulting from the competition between stable and unstable modes should appear near a phase transition line, since the first unstable modes appear there. Such behaviors can be incorpo- 
rated as biological functionality as shown in the few examples discussed in this letter, but this functionality has nothing to do with the notion of complexity "at the edge of chaos" and the hypothetical universal computational properties which may or may not exist there. 223, 25]

ER would like to thank the the Santa Fe Institute and the NSF REU program, and the UGS program at LANL which supported parts of this research.

\section{References}

[1] E. O. Wilson, The Insect Societies, Cambridge: Belknap Press, 1971; B. Hölldobler, and E. O. Wilson, The Ants, Cambridge: Belknap (1990).

[2] W. Hangartner, Z. vergl. Physiol. 57, 103 (1967).

[3] V. Calenbuhr and J.-L. Deneubourg, J. Theor. Biol. 158, 359 (1991).

[4] V. Calenbuhr, L. Chrétien, J.-L. Deneubourg and C. Detrain, J. Theor. Biol. 158, 395 (1991).

[5] R. Beckers, J.-L. Deneubourg, S. Goss. and J. M. Pasteels, Insectes Soc. 373, 258 (1990).

[6] J.-L. Deneubourg, S. Aron, S. Goss and J. M. Pasteels, J. Insect Behav. 32, 159 (1990).

[7] S. Goss, R. Beckers, J.-L. Deneubourg, S. Aron, and J. M. Pasteels, In: Behavioral Mechanisms of Food Selection, Nato ASI Series G20 (Hughes, ed.) Berlin Heidelberg: Springer-Verlag (1990).

[8] V. Calenbuhr and J.-L. Deneubourg, In:Biological Motion (W. Alt, and G. Hoffmann, eds.) 453, Berlin: Springer-Verlag (1990).

[9] M. M. Millonas, J. Theor. Biol. 159, 529 (1992).

[10] M. M. Millonas, In: Artificial Life III(ed. C. G. Langton). Santa Fe Institute Studies in the Sciences of Complexity, Proc. Vol XVII. Reading, Massachussetts: Addison-Wesley, (1994). 
[11] M. M. Millonas, In: Pattern Formation in Physical and Biological Sciences (ed. P. Claudis). Santa Fe Institute Studies in the Sciences of Complexity, Reading Massachussets: Addison-Wesley (1994).

[12] E. O. Wilson, Animal Behavior 10, 134-164 (1962).

[13] R. P. Evershed, E. D. Morgan and M. C. Cammaerts, Insect Biochem. 12, 383 (1981).

[14] C. Detrain, J. M. Pasteels, and J.-L. Deneubourg, Actes coll. Insectes Sociaux 4, 87 (1988).

[15] S. Gérardy and J. C. Verhaeghe, Actes coll. Insectes Sociaux 4, 235 (1988).

[16]

[17] L. Lam and R.D. Pochy, Compt. Phys. 7, 534 (1993); Pochy, D.R. Kayser, L.K. Aberle and L. Lam, Physica D 66, 166 (1993); F. Schweitzer and L. Schmansky-Geier, Physica A 206, 359 (1994).

[18] H. Haken, Synergetics, Berlin: Springer-Verlag (1983).

[19] S. A. Kauffman, J. Theor. Biol. 119 , 1 (1986).

[20] J. D. Farmer, S. A. Kauffman and N. H. Packard, Physica D 22, 50 (1986).

[21] R. Beckers, J.-L. Deneubourg and S. Goss, J. Theor. Biol. 159 , 397 (1992).

[22] P. Bak, C. Tang and K. Weisenfield, Phys. Rev. A 38, 364 (1988).

[23] N. H. Packard, In: Complexity in Biological Modeling (eds. S. Kelso and M. Shlesinger) (1988).

[24] P. Bak, K. Chen and M. Creutz, Nature 342, 780 (1989).

[25] C. G. Langton, Computation at the edge of chaos: Phase transitions and emergent computation, Ph. D. Thesis, University of Michigan, 1991. 
Figure 1: Trail formation. A: shows the swarm at time 29, when the field is still mostly random. B: at $t=208 \mathrm{C}$ : at $t=332$, the network has initially formed, $\mathrm{D}$ : at $t=412$. $\mathrm{E}$ : at $t=785$, where some branches have disappeared. $\mathrm{F}$ : at $t=1753$, shows the final network that emerges.

Figure 2: Physiological phase plot in $\delta-\gamma$ space for $\sigma_{0}=1.367$. Figure show the distribution of particles after 5000 time steps centered on the values of the physiological parameter for that run.

Figure 3: Memory erasure and reconsolidation. A: The original pattern. B: $\beta$ is turned back up after 250 time steps; the trail re-forms completely. $\mathrm{C}$ : $\beta$ is turned back up after 265 steps; only a portion of the trail remains. D: $\beta$ is turned back up after 275 steps. $\mathrm{E}$ : $\beta$ is turned back up after 300 steps. $\mathrm{F}: \beta$ is turned back up after 375 steps.

Figure 4: The "bootstrapping" effect. The Plot show the onset the effect of the introduction of a bootsrapping trail. Plot A shows the random configuration of the ants before the trail is added. B: shows the swarm in the short period of amplifying this weak trail. C: the final state. 